\title{
Comparative Study of Bachelor of Civil Engineering Curricula in Higher Learning Institutions in Tanzania: A Systematic Review
}

\author{
Dr. Yusuph B. Mhando ${ }^{1 *}$ Dr. Gislar E. Kifanyi ${ }^{2}$ \\ 1.Department of Civil Engineering, Arusha Technical College, Arusha, Tanzania \\ 2.Department of Construction Management and Technology, Mbeya University of Science and Technology, \\ Mbeya, Tanzania
}

\begin{abstract}
This paper compares the curricula of Bachelor degree in the field of civil engineering. This helps improving the quality of curriculum that generates demand driven engineering graduates with an equal competency in the context of labour market. Descriptive survey was adopted, and a checklist of courses from purposively selected higher learning institutions was used. Constant comparative analysis to categorize types of information investigated was adopted. Findings indicate that Bachelor degree programme in civil engineering at Dar es Salaam Institute of Technology (DIT), Mbeya University of Science and Technology (MUST) and St. Joseph College of Engineering and Technology (SJCET) has duration of 3 or 4 years depending on the entry qualification of the student, whereas at Arusha Technical College (ATC) it takes 3 years to complete the programme. On the other hand, inconsistency on the number of courses and academic loads offered in these institutions was revealed. The study suggests a joint development of curricula for training programmes. This could help trainers, researchers, curricula developers and policy makers to harmonize and minimize discrepancies of the curricula contents of the same programme offered in different institutions.
\end{abstract}

Keywords: curricula, engineers, bachelor degree, civil engineering, institutions, Tanzania

DOI: $10.7176 / \mathrm{JEP} / 12-29-05$

Publication date:October $31^{\text {st }} 2021$

\section{Introduction}

Civil engineering is the profession of designing and executing structural works that serves the general public, such as dams, bridges, aqueducts, canals, highways, power plants, chemical process plants, nuclear power stations, water desalination sewerage systems, transportation and other infrastructure (Davies and Marsh, 2021; Sutopo, 2018; Tabsh et al., 2012; Chen and Liew, 2003). The term was first used in the 18th century to distinguish the newly recognized profession from military engineering, until then preeminent (Davies and Marsh, 2021). From earliest times, however, engineers have engaged in peaceful activities, and many of the civil engineering works of ancient and medieval times such as the Roman public baths, roads, bridges, and aqueducts; the Flemish canals; the Dutch sea defenses; the French Gothic cathedrals; and many other monuments reveal a history of inventive genius and persistent experimentation (Davies and Marsh 2021). In this context, the functions of the civil engineer can be divided into three categories: those performed before construction (feasibility studies, site investigations, and design), those performed during construction (dealing with clients, consulting engineers, and contractors), and those performed after construction (maintenance and research) (Davies and Marsh, 2021). Thus, it is a matter of fact that, engineers play a key role in contributing to economic progress, enhance social and physical infrastructure, and inspire the changes that improve lives of people worldwide, particularly in developing countries.

However, only a qualified and competent workforce can carry the civil engineering of today to the future, turning it into a more innovative and competitive sector (Akyazi et al., 2020). On the other hand, the term 'curriculum' originated from the Greek word 'curere' meaning to run a course (Tinsae, 2016). According to Su (2012), curriculum seems to be considered greatly as what teachers are going to teach and, in other words, what learners are going to learn. In fact, "curriculum" is also closely related to how well the learners learn-the outcomes (Su, 2012). Thus, as an umbrella term, "curriculum" includes a lot of issues, for example, teaching curriculum, learning curriculum, testing curriculum, administrative curriculum and the hidden curriculum ( $\mathrm{Su}$, 2012). Furthermore, $\mathrm{Su}$ (2012) insists that curriculum can be seen as a means of achieving specific educational goals and objectives. According to Tinsae (2016) curriculum is defined as a combination whole including philosophical perspectives, the learner's and teacher's experience, instructional methodologies and expected and unexpected outputs acquired within a learning institution.

Moreover, curriculum can be defined as a plan of the teaching-learning process that students of an academic programme are required to undergo to achieve some specific objectives, and it includes scheme of studies, objectives and learning outcomes, course contents, teaching methodologies and assessment criteria. Nevertheless, debate is still continuing as to the definition of curriculum as it has varied definitions and this variation is due to scholars' position or approach or philosophical basis or understanding of the world in general (Tinsae, 2016). This study focuses on the curriculum of Bachelor of engineering degree in the field of civil engineering. As 
previous stated, it is pertinent to note that, engineering curriculum produces engineers who play a key role in contributing to economic progress, enhance social and physical infrastructure, and inspire the changes that improve lives of people globally.

It may be argued, in the sense that, the training of engineers requires continuous improvement to the content of the training curriculum that provides relevant skills and knowledge. According to Zhou et al. (2014), changes on modern life and social demand put forward more requirements to professional skills of engineers and technical personnel. Therefore, the civil engineering sector is a strategic sector to face the challenges of today's society, such as the climate emergency, the reduction of energy consumption and the sustainable use of resources (Akyazi et al., 2020). It is insisted that, the aim of education and training is to enable students to learn, while teaching has to be considered as a series of activities that stimulate, facilitate and progressively guide the learning process, culminating in a graduate who has the skills to engage in lifelong, self-directed, and reflective learning (Oliver et al., 2008). As this is, in fact, curriculum plays a central role towards production of skilled, competent and knowledgeable human resources to meet the demands of the country's social and economic development.

Thus, the comparison analysis of the features of the curricula for Bachelor of engineering in the field of civil engineering offered by the accredited tertiary higher learning institutions in the country is relevant. Accreditation agencies such as Tanzania Commissions for Universities (TCU) and the National Council for Technical Education (NACTE) both under the Ministry of Education, Science and Technology (MoEST) are responsible for regulating, advising and supporting engineering education to enhance its quality and to make the graduates national, regional and internationally competitive. The availability of high-quality training programmes will help to improve the quality of training, develop the processes of internationalization of the national educational system, expand and strengthen international educational and scientific ties (Eshniyazov et al., 2020). Furthermore, it is maintained that, accreditation agencies are responsible for, among others, facilitating engineering education to enhance its quality (Bhat et al., 2020).

As a matter of fact, NACTE obliges tertiary institutions to carry out a comprehensive self-study in every five years including curriculum review following initial accreditation (NACTE, 2001). Markedly, it is imperative periodically to evaluate and revise the status of the curriculum to address the need of the professional changing trends in the labour market. In this context, few Colleges and Universities in Tanzania have adopted a curriculum of Bachelor of engineering in the field of civil engineering. Typically, this curriculum is offered either through the College of Engineering and the Built Environment, College of Engineering or the Department of Civil Engineering. However, there is much multiplicity in the types of Bachelor of engineering courses in the field of civil engineering. Fortunately, several scientific studies were carried out on the comparison of training programmes in different countries. These previous studies include Eshniyazov et al. (2020) who found that, among others, there was larger volume of theoretical training of students in Ukraine while in Uzbekistan a larger amount of time is spent on obtaining practical skills during all types of practices. Shuaibu et al. (2019) compared the curricula structure similarities and differences in Malaysia and Nigeria, and found that the courses contained in the curricula are similar professionally except the presence of general courses. Other researchers were Tabsh et al. (2012) who managed to find out that in Arab countries curricula took either 4 or 5 years to complete and the number of credit hours were found to be as little as 131 and as large as 204 credits. Ebadi et al. (2020) managed to categorize and compare curricula from several Canadian universities and found that some universities paid less attention to a specific stream in comparison with the average, which could be identified as a gap in the curriculum.

Markedly, while those previous studies were able to draw reasonable conclusions, indeed, they were not sufficient to describe and compare the contents of curricula for Bachelor of engineering in the field of civil engineering offered by different higher learning institutions. Interestingly, though some relevant literatures are available, there has been no such comparative study of curricula of Bachelor of engineering in the field of civil engineering in Tanzania. Furthermore, it is determined that, civil engineering undergraduate degree courses last for three or four years with two (15-teaching weeks x 2) semesters per year. Few optional courses are offered at the end of the program for students to deepen their knowledge and skills in certain specific areas such as structures, reinforced concrete design, transportation, water resources and geotechnical engineering (Arusha Technical College (ATC), 2021; Dar es Salaam Institute of Technology (DIT), 2021; Mbeya University of Science and Technology (MUST), 2021; St. Joseph College of Engineering and Technology (SJCET), 2021; Ebadi et al., 2020; Eshniyazov et al., 2020; Liu and Siddharthan, 2016; Zhou et al. 2014; Tabsh et al., 2012; Tapia and Najafi, 2003).

Besides, students are normally encouraged to focus in specific areas through their final project work (ATC, 2021; DIT, 2021; MUST, 2021; SJCET, 2021; Ebadi et al., 2020; Eshniyazov et al., 2020; Liu and Siddharthan, 2016; Zhou et al. 2014; Tabsh et al., 2012; Tapia and Najafi, 2003). As earlier stated, Bachelor degree programme of civil engineering consists 6 or 8 semesters depending on the entry qualifications (Full Technician Certificate (FTC), Diploma or form six qualifications) with an average of seven modules per semester. In the 
first four semesters, that is first year and second year, all students are required to take fundamental modules including calculus, communication skills, differential equations and complex variables (ATC, 2020; DIT, 2020; MUST, 2020; SJCET, 2020; Ebadi et al., 2020; Eshniyazov et al., 2020; Liu and Siddharthan, 2016; Zhou et al. 2014; Tabsh et al., 2012; Tapia and Najafi, 2003); and general courses such as engineering drawing, construction technology, soil mechanics and structural mechanics for those who have form six qualifications. The remaining two years or one year are dedicated to the engineering sciences modules such as mechanics of solids, static and dynamics, and professional modules such as bridge design, reinforced concrete design, timber and steel design, foundation design, transportation engineering and ground water engineering respectively (ATC, 2020; DIT, 2020; MUST, 2020; SJCET, 2020; Ebadi et al., 2020; Eshniyazov et al., 2020; Liu and Siddharthan, 2016; Zhou et al. 2014; Tabsh et al., 2012; Tapia and Najafi, 2003).

This study compares curricula for training Bachelor of engineering students in the field of civil engineering adopted by MUST, DIT, ATC and SJCET of the St. Joseph University in Tanzania (SJUIT). Specifically, this study was to: identify the differences and similarities of the curricula in terms of general features, objectives and contents; and to develop recommendations for improving the training programme. In fact, this could help relevant stakeholders such as trainers, researchers, curricula reviewers, the industry and policy-makers to enhance the quality of curricula that generate demand-driven engineering graduates with an equal competency in the context of the labour market.

\section{Materials and Methods}

\subsection{Research Design}

The research design of the study was solely a descriptive survey conducted to identify the differences and similarities of the curricula for Bachelor of engineering in the field of civil engineering for improvement. Creswell (2009) clarifies that research designs are plans and procedures for research that span the decisions from broad assumptions to detailed methods of data collection and analysis. Further, the study adopted methods of comparison, analysis, synthesis and logical generalization (Eshniyazov et al., 2020) to determine the differences and similarities of the curricula in terms of general features, objectives and contents. Specifically, this paper focused on the curricula of Bachelor of engineering in the field of civil engineering offered to undergraduate students at Mbeya University of Science and Technology (MUST), Dar es Salaam Institute of Technology (DIT), Arusha Technical College (ATC) and ST. Joseph College of Engineering and Technology (SJCET) of the St. Joseph University in Tanzania (SJUIT). The minimum academic standard programmes (curricula) accredited by regulatory bodies such as TCU and NACTE adopted in Universities and Colleges to offer Bachelor of engineering programmes were used as the checklists. The respective contents of Bachelor of engineering curricula run by four higher learning institutions were critically looked into, in relation to the respective minimum standards (Nwosu et al., 2013).

\subsection{Data Analysis}

Data were collected from online curriculum (Ebadi et al., 2020) of each programme contained in the prospectus of each involved higher learning institution. The analysis outlined the common areas of education and points of diversion among the civil engineering curricula (Tabsh et al., 2012). The number of credit hours in each of the courses in the curricula were also critically analyzed and discussed. For clarity, the results of analysis were presented using Tables and histograms. Arguably, comparative research is suitable for both qualitative and quantitative methods as advocated by William in Serçe and Acar (2021).

\subsection{Research Framework}

The identified dependent variable in this study was the curricula contents of Bachelor of engineering in the field of civil engineering offered at DIT, ATC, MUST and SJCET. The independent variables in this scientific study were the differences and similarities of the compared curricula in terms of general features, objectives and contents.

\section{Results and Discussion}

Results and discussion of the study were based on the analysis of the curricula of Bachelor of engineering in the field of civil engineering. These four institutions (DIT, ATC, MUST and SJCET) are accredited higher learning tertiary institutions in the country that offer Bachelor of engineering programmes in the field of civil engineering. Investigation shows that all considered civil engineering programmes offered in these four institutions were based on the semester system. In that case, Bachelor of civil engineering programme consists 6 or 8 semesters depending on the entry qualifications (Full Technician Certificate (FTC), Diploma or form six qualifications) with an average of seven modules per semester. Interestingly, all four prestigious higher learning institutions in the country offer Diploma (NTA Level 6), Higher National Diploma (HND) (NTA Level 7) and Bachelor of Engineering degree (NTA Level 8) respectively in the field of civil engineering. Furthermore, the institutions 
offer curricula that allow a student to exit with HND (NTA Level 7) when completing second year or third year, depending on the duration ( 3 or 4 years) of the Bachelor degree programme. In fact, DIT was established by the Act of Parliament No.6 of 1997 as a higher technical training institution in Tanzania (DIT, 2021). ATC was granted an autonomy under Establishment Order No. 78 enabled by NACTE Act No. 9 of 1997, and the College was established in 1978 (ATC, 2021). In short, MUST was a result of the transformation of Mbeya Institute of Science and Technology (MIST) through the University Act No. 7 of 2005 and the Charter of Mbeya University of Science and Technology 2013 (MUST, 2021), while SJCET is one of the Colleges of St. Joseph University in Tanzania (SJUIT). SJUIT is a full-fledged private University accredited by the Tanzania Commission for Universities (TCU) in its Order dated 21st December 2011(SJCET, 2021). All these four higher learning institutions are under the Ministry of Education Science and Technology (MoEST) of Tanzania. The courses for the programmes offered in these institutions include general courses, fundamental courses, core courses and elective courses. In that case, students are required to dedicate number of courses and number of credits hours needed to complete the programme of study.

\subsection{Scheme of Study}

Normally the definition of "credit" varies from one institution to another, depending on the allocated hours per week for a student to undergo training. According to Tapia and Najafi (2003) credit could be defined as the one semester hour, generally representing one hour (50-minute) per week of lecture or two or more hours per week of laboratory work. On the other hand, credits are the total hours that students attend a course, which is set by the institution. As maintained by Tapia and Najafi (2003), the curricula of the institutions (DIT, ATC, MUST and SJCET) adopted the following definition of total credits per course:

$$
\mathrm{C}=\mathrm{L}+\mathrm{T}+\mathrm{P}+\mathrm{A}
$$

Where C, L, T, P and A are total credits for the course, number of 45-minutes lectures, number of 45-minutes tutorials, number of 45-minutes practical class, number of 45-minutes assignment class respectively. For instance, a reinforced concrete design module taught at ATC usually has 4 hours (1hour lecture, 1hour tutorial, 0hour practical and 2 hours assignment) with 6 credits. So, students are required to dedicate at least four hours for this course.

\subsection{General Courses}

Result in Table1 shows the extent of differences and similarities of the general courses. DIT has 24 general courses with 173 credits constituting $57.14 \%$ of the total 42 identified general courses. MUST has 18 general courses and 120 credits constituting $42.86 \%$. SJCET has 15 general courses and 180 credits constituting $35.71 \%$. In this case, ATC has zero general courses constituting $0 \%$ of the total 42 identified general courses. Arguably, this is because ATC does not admit students who have form six qualifications in its Bachelor programme in the field of civil engineering. However, the total number of courses and the total number of credits hours needed for completing general courses varied significantly from one institution to another. Tabsh at el. (2012) found that the total number of credits hours needed for completing the degree in the Arab world varied significantly from one country to another, and even within the same country. In fact, this scenario could cause inconsistency of the skills and knowledge inculcated to a student from one institution to another offering the same engineering degree programme.

Table 1. General Courses

\begin{tabular}{|c|c|c|c|c|c|c|c|c|c|}
\hline \multirow[b]{3}{*}{ SN } & \multirow[b]{3}{*}{ General courses } & \multicolumn{8}{|c|}{ Institutions } \\
\hline & & \multicolumn{2}{|c|}{ DIT } & \multicolumn{2}{|c|}{ ATC } & \multicolumn{2}{|c|}{ MUST } & \multicolumn{2}{|c|}{ SJCET } \\
\hline & & 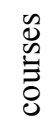 & $\begin{array}{l}\stackrel{0}{0} \\
\stackrel{0}{0} \\
\stackrel{0}{0}\end{array}$ & $\begin{array}{l}\text { d } \\
\text { के } \\
\tilde{\Xi}\end{array}$ & 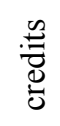 & $\begin{array}{l}0 \\
0 \\
\vdots \\
0 \\
0\end{array}$ & 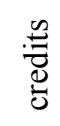 & 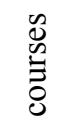 & 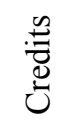 \\
\hline 1 & Engineering drawing I & $\sqrt{ }$ & 9 & - & - & $\sqrt{ }$ & 9 & $\sqrt{ }$ & 12 \\
\hline 2 & Construction technology I & $\sqrt{ }$ & 12 & - & - & $\sqrt{ }$ & 12 & - & - \\
\hline 3 & Building construction I & $\sqrt{ }$ & 9 & - & - & $\sqrt{ }$ & 9 & - & - \\
\hline 4 & Soil mechanics & $\sqrt{ }$ & 15 & $\begin{array}{l}- \\
\end{array}$ & - & $\sqrt{ }$ & 6 & - & - \\
\hline 5 & Structural mechanics I & $\sqrt{ }$ & 5 & - & - & $\sqrt{ }$ & 3 & - & - \\
\hline 6 & Land/linear surveying I & $\sqrt{ }$ & 9 & - & - & $\sqrt{ }$ & 6 & - & - \\
\hline 7 & Civil engineering materials I & $\sqrt{ }$ & 6 & 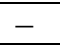 & - & $\sqrt{ }$ & 6 & - & - \\
\hline 8 & $\begin{array}{l}\text { Road construction and } \\
\text { maintenance I }\end{array}$ & $\sqrt{ }$ & 12 & - & - & $\sqrt{ }$ & 4 & - & - \\
\hline 9 & Engineering drawing II & - & - & - & - & $\sqrt{ }$ & 6 & - & - \\
\hline 10 & Construction technology II & - & - & 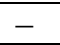 & - & $\sqrt{ }$ & 6 & - & - \\
\hline
\end{tabular}




\begin{tabular}{|c|c|c|c|c|c|c|c|c|c|}
\hline \multirow[b]{3}{*}{$\mathrm{SN}$} & \multirow[b]{3}{*}{ General courses } & \multicolumn{8}{|c|}{ Institutions } \\
\hline & & \multicolumn{2}{|l|}{ DIT } & \multicolumn{2}{|c|}{ ATC } & \multicolumn{2}{|l|}{ MUST } & \multicolumn{2}{|l|}{ SJCET } \\
\hline & & $\begin{array}{l}\mathscr{U} \\
\stackrel{0}{0} \\
\tilde{\delta}\end{array}$ & : & $\begin{array}{l}\mathscr{E} \\
\stackrel{\omega}{\Xi} \\
0\end{array}$ & $\begin{array}{l}: \stackrel{0}{0} \\
\stackrel{0}{0} \\
0\end{array}$ & $\begin{array}{l}\mathscr{U} \\
\stackrel{\omega}{\Xi} \\
\dot{0}\end{array}$ & $\begin{array}{l}\stackrel{\mathscr{Z}}{0} \\
\stackrel{0}{0} \\
0\end{array}$ & 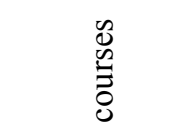 & :气 \\
\hline 11 & Building construction II & - & - & - & - & $\sqrt{ }$ & 6 & - & - \\
\hline 12 & $\begin{array}{l}\text { Structural } \\
\text { mechanics/analysis }\end{array}$ & $\sqrt{ }$ & 9 & - & - & $\sqrt{ }$ & 3 & - & - \\
\hline 13 & Land surveying II & - & - & - & - & $\sqrt{ }$ & 6 & - & - \\
\hline 14 & $\begin{array}{l}\text { Civil engineering materials } \\
\text { II }\end{array}$ & - & - & - & - & $\sqrt{ }$ & 6 & - & - \\
\hline 15 & $\begin{array}{l}\text { Road construction and } \\
\text { maintenance II }\end{array}$ & - & - & - & - & $\sqrt{ }$ & 8 & - & - \\
\hline 16 & Water supply and sanitation & - & - & - & - & $\sqrt{ }$ & 6 & - & - \\
\hline 17 & Workshop practice & - & - & - & - & $\sqrt{ }$ & 8 & $\sqrt{ }$ & 9 \\
\hline 18 & Industrial practical training & $\sqrt{ }$ & 10 & - & - & $\sqrt{ }$ & 10 & - & - \\
\hline 19 & $\begin{array}{l}\text { Computer basics and word } \\
\text { processing }\end{array}$ & $\sqrt{ }$ & 2 & - & - & - & - & $\sqrt{ }$ & 9 \\
\hline 20 & $\begin{array}{l}\text { Electrical installation and } \\
\text { drafting }\end{array}$ & $\sqrt{ }$ & 12 & - & - & - & - & $\sqrt{ }$ & 12 \\
\hline 21 & $\begin{array}{l}\text { Basic technical } \\
\text { communication skills }\end{array}$ & $\sqrt{ }$ & 2 & - & - & - & - & - & - \\
\hline 22 & $\begin{array}{l}\text { Entrepreneurship concepts } \\
\text { and context }\end{array}$ & $\sqrt{ }$ & 3 & - & - & - & - & - & - \\
\hline 23 & Gas welding process & $\sqrt{ }$ & 9 & - & - & - & - & - & - \\
\hline 24 & Static and dynamics & $\sqrt{ }$ & 3 & - & - & - & - & - & - \\
\hline 25 & Labour based technology & $\sqrt{ }$ & 9 & - & - & - & - & - & - \\
\hline 26 & Spreadsheet and database & $\sqrt{ }$ & 2 & - & - & - & - & - & - \\
\hline 27 & Communication skills & $\sqrt{ }$ & 2 & - & - & - & - & $\sqrt{ }$ & 12 \\
\hline 28 & Small business development & $\sqrt{ }$ & 3 & - & - & - & - & - & - \\
\hline 29 & Welding processes & $\sqrt{ }$ & 9 & - & - & - & - & - & - \\
\hline 30 & $\begin{array}{l}\text { Gravitation and simple } \\
\text { harmonic motion }\end{array}$ & $\sqrt{ }$ & 3 & - & - & - & - & - & - \\
\hline 31 & $\begin{array}{l}\text { Introduction to architectural } \\
\text { drawing }\end{array}$ & $\sqrt{ }$ & 9 & - & - & - & - & - & - \\
\hline 32 & $\begin{array}{l}\text { Maintenance and } \\
\text { construction techniques }\end{array}$ & $\sqrt{ }$ & 9 & - & - & - & - & - & - \\
\hline 33 & Engineering mathematics I & - & - & - & - & - & - & $\sqrt{ }$ & 15 \\
\hline 34 & Engineering physics & - & - & - & - & - & - & $\sqrt{ }$ & 15 \\
\hline 35 & Basic civil engineering & - & - & - & - & - & - & $\sqrt{ }$ & 12 \\
\hline 36 & $\begin{array}{l}\text { Basic mechanical } \\
\text { engineering }\end{array}$ & - & - & - & - & - & - & $\sqrt{ }$ & 12 \\
\hline 37 & Engineering mechanics & - & - & - & - & - & - & $\sqrt{ }$ & 12 \\
\hline 38 & Engineering mathematics II & - & - & - & - & - & - & $\sqrt{ }$ & 15 \\
\hline 39 & Computer programming & - & - & - & - & - & - & $\sqrt{ }$ & 12 \\
\hline 40 & $\begin{array}{l}\text { basic electronics } \\
\text { engineering }\end{array}$ & - & - & - & - & - & - & $\sqrt{ }$ & 12 \\
\hline 41 & $\begin{array}{l}\text { Environmental science and } \\
\text { engineering }\end{array}$ & - & - & - & - & - & - & $\sqrt{ }$ & 12 \\
\hline 42 & $\begin{array}{l}\text { Computer programming } \\
\text { laboratory }\end{array}$ & - & - & - & - & - & - & $\sqrt{ }$ & 9 \\
\hline & Total /Percentage & $24(57.14 \%)$ & 173 & $0(0 \%)$ & 0 & $18(42.86 \%)$ & 120 & $15(35.71 \%)$ & 180 \\
\hline
\end{tabular}

\subsection{Fundamental Courses for HND}

Result in Table 2 reveals the extent of differences and similarities of the fundamental courses offered at four sampled higher learning institutions (DIT, ATC, MUST and SJCET). In this case, DIT has 9 fundamental 
courses with 57 credits constituting $64.29 \%$ of the total 14 identified fundamental courses. ATC has 6 fundamental courses constituting $42.86 \%$ with 36 credits. MUST has 8 fundamental courses constituting $57.14 \%$ with 46 credits. SJCET has 3(21.43\%) fundamental courses with 27 credits. Therefore, it can be argued that, DIT has more fundamental courses for HND followed by MUST and ATC respectively. However, SJCET has low number (3) of fundamental courses. Interestingly, students for engineering degree courses at these institutions may exit at NTA level 7 and awarded a Higher National Diploma (HND). Nwosu et al. (2013) affirm that two federal polytechnics in Nigeria offer Higher National Diploma (HND) in library and information sciences. Furthermore, the results show inconsistency on the number of fundamental courses and credits offered by the investigated institutions. Ebadi et al. (2020) compared the curricula of different Canadian universities and found that discrepancies exist between programmes, especially in number of courses offered.

Table 2. Fundamental Courses for HND

\begin{tabular}{|c|c|c|c|c|c|c|c|c|c|}
\hline \multirow[b]{3}{*}{$\mathrm{SN}$} & \multirow[b]{3}{*}{ Fundamental courses } & \multicolumn{8}{|c|}{ Institutions } \\
\hline & & \multicolumn{2}{|c|}{ DIT } & \multicolumn{2}{|c|}{ ATC } & \multicolumn{2}{|l|}{ MUST } & \multicolumn{2}{|l|}{ SJCET } \\
\hline & & $\begin{array}{l}\tilde{d} \\
\omega \\
\vdots \\
\delta\end{array}$ & 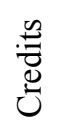 & $\begin{array}{l}\mathscr{W} \\
\stackrel{\mathscr{E}}{0} \\
0\end{array}$ & $\begin{array}{l}\stackrel{\mathscr{Z}}{0} \\
\stackrel{0}{0}\end{array}$ & $\begin{array}{l}\mathscr{d} \\
\stackrel{\infty}{\Xi} \\
0\end{array}$ & 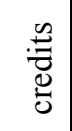 & $\begin{array}{l}0 \\
0 \\
\vdots \\
0 \\
0\end{array}$ & 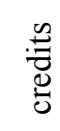 \\
\hline 1 & Advanced calculus & $\sqrt{ }$ & 6 & $\sqrt{ }$ & 6 & $\sqrt{ }$ & 6 & - & - \\
\hline 2 & Communication skills & $\sqrt{ }$ & 6 & $\sqrt{ }$ & 6 & $\sqrt{ }$ & 4 & - & - \\
\hline 3 & $\begin{array}{l}\text { Computing using } \\
\text { mathematical software }\end{array}$ & $\sqrt{ }$ & 6 & - & - & - & - & - & - \\
\hline 4 & $\begin{array}{l}\text { Data structure computer } \\
\text { programming }\end{array}$ & $\sqrt{ }$ & 9 & $\sqrt{ }$ & 6 & $\sqrt{ }$ & 6 & - & - \\
\hline 5 & $\begin{array}{l}\text { Differential equations and } \\
\text { complex variables }\end{array}$ & $\sqrt{ }$ & 6 & $\sqrt{ }$ & 6 & $\sqrt{ }$ & 6 & - & - \\
\hline 6 & Engineering mathematics III & - & - & - & - & - & - & $\sqrt{ }$ & 9 \\
\hline 7 & Entrepreneurship education I & - & - & - & - & $\sqrt{ }$ & 6 & - & - \\
\hline 8 & Linear algebra and calculus & $\sqrt{ }$ & 6 & $\sqrt{ }$ & 6 & $\sqrt{ }$ & 6 & - & - \\
\hline 9 & Numerical methods & - & - & - & - & - & - & $\sqrt{ }$ & 9 \\
\hline 10 & Object oriented programming & $\sqrt{ }$ & 6 & - & - & $\sqrt{ }$ & 6 & - & - \\
\hline 11 & Operational research & - & - & - & - & - & - & $\sqrt{ }$ & 9 \\
\hline 12 & Probability and statistics & $\sqrt{ }$ & 6 & - & - & - & - & - & - \\
\hline 13 & $\begin{array}{l}\text { Research methods for } \\
\text { engineers }\end{array}$ & $\sqrt{ }$ & 6 & - & - & - & - & - & - \\
\hline 14 & $\begin{array}{l}\text { Statistics and numerical } \\
\text { analysis }\end{array}$ & - & - & $\sqrt{ }$ & 6 & $\sqrt{ }$ & 6 & - & - \\
\hline & Total /Percentage & $9(64.29 \%)$ & 57 & $6(42.86 \%)$ & 36 & $8(57.14 \%)$ & 46 & $3(21.43 \%)$ & 27 \\
\hline
\end{tabular}

3.4 Core Courses for HND

Table 3 shows the comparison of the curricula contents. As a result, SJCET has more courses 30(40.54\%) and 281 credits of the total 74 core courses for HND as compared to other institutions. DIT has 29(39.19\%) core courses with 192 credits and, MUST has 28 core courses constituting 37.84\% with 197 credits. However, ATC has $27(36.49 \%$ ) core courses with 198 credits. In fact, the study result of curricula comparison shows inconsistency on the number of core courses offered. Evidently, Ebadi et al. (2020) compared the curricula of different Canadian universities and found that discrepancies exist between programmes, especially in number of courses offered. Furthermore, Tapia and Najafi (2003) argue that the major aspects taken into account in the curricula comparison were the number of courses of each curriculum and the differences in the student academic load. 
Table 3. Core Courses for HND

\begin{tabular}{|c|c|c|c|c|c|c|c|c|c|}
\hline \multirow{3}{*}{$\begin{array}{c}\mathrm{SN} \\
1\end{array}$} & \multirow{3}{*}{$\begin{array}{l}\text { Core courses/modules } \\
\begin{array}{l}\text { Analysis of crop water } \\
\text { requirement }\end{array}\end{array}$} & \multicolumn{8}{|c|}{ Institutions } \\
\hline & & \multicolumn{2}{|c|}{ DIT } & \multicolumn{2}{|c|}{ ATC } & \multicolumn{2}{|c|}{ MUST } & \multicolumn{2}{|c|}{ SJCET } \\
\hline & & - & - & $\sqrt{ }$ & 6 & - & - & - & - \\
\hline 2 & $\begin{array}{l}\text { Applied hydraulic } \\
\text { engineering }\end{array}$ & - & - & - & - & - & - & $\sqrt{ }$ & 9 \\
\hline 3 & Architecture & - & - & - & - & - & - & $\sqrt{ }$ & 9 \\
\hline 4 & Basic structural theory & $\sqrt{ }$ & 6 & - & - & - & - & - & - \\
\hline 5 & Building construction & $\sqrt{ }$ & 6 & - & - & - & - & - & - \\
\hline 6 & $\begin{array}{l}\text { Building planning and } \\
\text { drawing }\end{array}$ & $\sqrt{ }$ & 6 & - & - & $\sqrt{ }$ & 6 & - & - \\
\hline 7 & Building science & - & - & - & - & - & - & $\sqrt{ }$ & 9 \\
\hline 8 & $\begin{array}{l}\text { Civil engineering } \\
\text { materials I }\end{array}$ & $\sqrt{ }$ & 6 & $\sqrt{ }$ & 9 & $\sqrt{ }$ & 6 & - & - \\
\hline 9 & $\begin{array}{l}\text { Civil engineering } \\
\text { materials II }\end{array}$ & $\sqrt{ }$ & 6 & - & - & $\sqrt{ }$ & 3 & - & - \\
\hline 10 & $\begin{array}{l}\text { Computer aided } \\
\text { building drawing }\end{array}$ & - & - & - & - & - & - & $\sqrt{ }$ & 9 \\
\hline 11 & $\begin{array}{l}\text { Computer aided } \\
\text { design (CAD) }\end{array}$ & - & - & $\sqrt{ }$ & 6 & - & - & - & - \\
\hline 12 & Concrete technology & $\sqrt{ }$ & 6 & - & - & $\sqrt{ }$ & 6 & $\sqrt{ }$ & 9 \\
\hline 13 & $\begin{array}{l}\text { Construction and } \\
\text { irrigation equipment }\end{array}$ & $\sqrt{ }$ & 6 & - & - & - & - & - & - \\
\hline 14 & $\begin{array}{l}\text { Construction } \\
\text { management }\end{array}$ & $\sqrt{ }$ & 9 & - & - & $\sqrt{ }$ & 6 & - & - \\
\hline 15 & $\begin{array}{l}\text { Construction of } \\
\text { multistory structures }\end{array}$ & $\sqrt{ }$ & 9 & - & - & - & - & - & - \\
\hline 16 & $\begin{array}{l}\text { Construction } \\
\text { technology }\end{array}$ & - & - & $\sqrt{ }$ & 4 & $\sqrt{ }$ & 6 & - & - \\
\hline 17 & $\begin{array}{l}\text { Contract planning and } \\
\text { administration }\end{array}$ & $\sqrt{ }$ & 6 & - & - & $\sqrt{ }$ & 4 & - & - \\
\hline 18 & $\begin{array}{l}\text { Design of diversion } \\
\text { and impounding } \\
\text { structures }\end{array}$ & - & - & $\sqrt{ }$ & 6 & - & - & - & - \\
\hline 19 & $\begin{array}{l}\text { Design of drainage } \\
\text { systems }\end{array}$ & - & - & $\sqrt{ }$ & 6 & - & - & - & - \\
\hline 20 & $\begin{array}{l}\text { Design of gravity flow } \\
\text { irrigation systems }\end{array}$ & - & - & $\sqrt{ }$ & 9 & - & - & - & - \\
\hline 21 & $\begin{array}{l}\text { Design of pressurized } \\
\text { irrigation systems }\end{array}$ & - & - & $\sqrt{ }$ & 6 & - & - & - & - \\
\hline 22 & Engineering geology & $\sqrt{ }$ & 9 & - & - & $\sqrt{ }$ & 4 & - & - \\
\hline 23 & $\begin{array}{l}\text { Engineering } \\
\text { hydrology }\end{array}$ & $\sqrt{ }$ & 6 & $\sqrt{ }$ & 6 & $\sqrt{ }$ & 4 & - & - \\
\hline 24 & Engineering survey I & $\sqrt{ }$ & 6 & $\sqrt{ }$ & 12 & $\sqrt{ }$ & 9 & $\sqrt{ }$ & 9 \\
\hline 25 & Engineering survey II & $\sqrt{ }$ & 9 & - & - & $\sqrt{ }$ & 9 & $\sqrt{ }$ & 9 \\
\hline 26 & $\begin{array}{l}\text { Environmental } \\
\text { engineering I }\end{array}$ & - & - & - & - & - & - & $\sqrt{ }$ & 9 \\
\hline 27 & $\begin{array}{l}\text { Environmental } \\
\text { engineering II }\end{array}$ & - & - & - & - & - & - & $\sqrt{ }$ & 9 \\
\hline 28 & Fluid mechanics & $\sqrt{ }$ & 6 & - & - & $\sqrt{ }$ & 9 & $\sqrt{ }$ & 9 \\
\hline 29 & $\begin{array}{l}\text { Fluid mechanics and } \\
\text { hydraulics }\end{array}$ & - & - & - & - & $\sqrt{ }$ & 9 & $\sqrt{ }$ & 9 \\
\hline 30 & $\begin{array}{l}\text { Foundation } \\
\text { engineering }\end{array}$ & - & - & - & - & $\sqrt{ }$ & 9 & - & - \\
\hline 31 & $\begin{array}{l}\text { Foundation } \\
\text { engineering }\end{array}$ & - & - & - & - & - & - & $\sqrt{ }$ & 9 \\
\hline
\end{tabular}




\begin{tabular}{|c|c|c|c|c|c|c|c|c|c|}
\hline \multirow{3}{*}{$\begin{array}{l}\mathrm{SN} \\
32\end{array}$} & \multirow{3}{*}{$\begin{array}{l}\text { Core courses/modules } \\
\text { Geometric design and } \\
\text { traffic engineering }\end{array}$} & \multicolumn{8}{|c|}{ Institutions } \\
\hline & & \multicolumn{2}{|c|}{ DIT } & \multicolumn{2}{|c|}{ ATC } & \multicolumn{2}{|c|}{ MUST } & \multicolumn{2}{|c|}{ SJCET } \\
\hline & & - & - & - & - & $\sqrt{ }$ & 9 & - & - \\
\hline 33 & $\begin{array}{l}\text { Geotechnical } \\
\text { engineering }\end{array}$ & - & - & $\sqrt{ }$ & 9 & - & - & - & - \\
\hline 34 & $\begin{array}{l}\text { Highway engineering } \\
\text { materials }\end{array}$ & - & - & - & - & $\sqrt{ }$ & 6 & $\sqrt{ }$ & 9 \\
\hline 35 & $\begin{array}{l}\text { Human resources } \\
\text { management }\end{array}$ & - & - & $\sqrt{ }$ & 6 & - & - & - & - \\
\hline 36 & $\begin{array}{l}\text { Industrial practical } \\
\text { training }\end{array}$ & $\sqrt{ }$ & 12 & $\sqrt{ }$ & 24 & $\sqrt{ }$ & 20 & $\sqrt{ }$ & 20 \\
\hline 37 & $\begin{array}{l}\text { Introduction to } \\
\text { metrology }\end{array}$ & - & - & $\sqrt{ }$ & 6 & - & - & - & - \\
\hline 38 & $\begin{array}{l}\text { Introduction to soil } \\
\text { science }\end{array}$ & - & - & $\sqrt{ }$ & 9 & - & - & - & - \\
\hline 39 & Irrigation engineering & - & - & - & - & - & - & $\sqrt{ }$ & 9 \\
\hline 40 & $\begin{array}{l}\text { Irrigation system } \\
\text { performance } \\
\text { evaluation }\end{array}$ & - & - & $\sqrt{ }$ & 6 & - & - & - & - \\
\hline 41 & $\begin{array}{l}\text { Irrigation water } \\
\text { quality }\end{array}$ & - & - & $\sqrt{ }$ & 6 & - & - & - & - \\
\hline 42 & $\begin{array}{l}\text { Labour based road } \\
\text { engineering }\end{array}$ & $\sqrt{ }$ & 3 & - & - & - & - & - & - \\
\hline 43 & $\begin{array}{l}\text { Measurement of } \\
\text { building and civil } \\
\text { works }\end{array}$ & $\sqrt{ }$ & 6 & - & - & - & - & - & - \\
\hline 44 & $\begin{array}{l}\text { Measurement of civil } \\
\text { and irrigation works }\end{array}$ & - & - & $\sqrt{ }$ & 6 & - & - & - & - \\
\hline 45 & Mechanics of solids & - & - & - & - & - & - & $\sqrt{ }$ & 9 \\
\hline 46 & $\begin{array}{l}\text { Mechanics of } \\
\text { structures }\end{array}$ & - & - & - & - & $\sqrt{ }$ & 4 & - & - \\
\hline 47 & Mini project & - & - & - & - & - & - & $\sqrt{ }$ & 9 \\
\hline 48 & $\begin{array}{l}\text { Open channel } \\
\text { hydraulics }\end{array}$ & $\sqrt{ }$ & 6 & - & - & - & - & - & - \\
\hline 49 & $\begin{array}{l}\text { Pavement design and } \\
\text { constr. of access roads }\end{array}$ & - & - & $\sqrt{ }$ & 9 & $\sqrt{ }$ & 9 & - & - \\
\hline 50 & $\begin{array}{l}\text { Quality control } \\
\text { management }\end{array}$ & - & - & $\sqrt{ }$ & 6 & - & - & - & - \\
\hline 51 & Quantity surveying I & $\sqrt{ }$ & 6 & - & - & $\sqrt{ }$ & 6 & - & - \\
\hline 52 & Quantity surveying II & - & - & - & - & $\sqrt{ }$ & 6 & - & - \\
\hline 53 & Rain water harvesting & - & - & $\sqrt{ }$ & 4 & - & - & - & - \\
\hline 54 & $\begin{array}{l}\text { Reinforced concrete } \\
\text { design and detailing I }\end{array}$ & $\sqrt{ }$ & 6 & - & - & $\sqrt{ }$ & 9 & - & - \\
\hline 55 & $\begin{array}{l}\text { Reinforced concrete } \\
\text { design and detailing II }\end{array}$ & $\sqrt{ }$ & 6 & - & - & $\sqrt{ }$ & 9 & - & - \\
\hline 56 & $\begin{array}{l}\text { Remote sensing and } \\
\text { GIS }\end{array}$ & - & - & $\sqrt{ }$ & 7 & - & - & $\sqrt{ }$ & 9 \\
\hline 57 & $\begin{array}{l}\text { Research } \\
\text { methodology }\end{array}$ & - & - & $\sqrt{ }$ & 3 & $\sqrt{ }$ & 4 & - & - \\
\hline 58 & Reservoir operation & - & - & $\sqrt{ }$ & 6 & - & - & - & - \\
\hline 59 & Route design & $\sqrt{ }$ & 6 & - & - & - & - & - & - \\
\hline 60 & Soil mechanics & $\sqrt{ }$ & 6 & $\sqrt{ }$ & 9 & $\sqrt{ }$ & 9 & $\sqrt{ }$ & 9 \\
\hline 61 & $\begin{array}{l}\text { Soil } \\
\text { technology/engineerin } \\
\text { g laboratory }\end{array}$ & $\sqrt{ }$ & 6 & - & - & - & - & $\sqrt{ }$ & 9 \\
\hline 62 & Strength of materials & $\sqrt{ }$ & 6 & - & - & - & - & $\sqrt{ }$ & 9 \\
\hline
\end{tabular}




\begin{tabular}{|c|c|c|c|c|c|c|c|c|c|}
\hline \multirow{3}{*}{$\begin{array}{l}\mathrm{SN} \\
63\end{array}$} & \multirow{3}{*}{$\begin{array}{l}\text { Core courses/modules } \\
\begin{array}{l}\text { Strength of materials } \\
\text { laboratory }\end{array}\end{array}$} & \multicolumn{8}{|l|}{ Institutions } \\
\hline & & \multicolumn{2}{|l|}{ DIT } & \multicolumn{2}{|l|}{ ATC } & \multicolumn{2}{|l|}{ MUST } & \multicolumn{2}{|l|}{ SJCET } \\
\hline & & - & - & - & - & - & - & $\sqrt{ }$ & 9 \\
\hline 64 & Structural analysis I & $\sqrt{ }$ & 6 & $\sqrt{ }$ & 6 & $\sqrt{ }$ & 4 & $\sqrt{ }$ & 9 \\
\hline 65 & Structural analysis II & - & - & - & - & $\sqrt{ }$ & 6 & $\sqrt{ }$ & 9 \\
\hline 66 & $\begin{array}{l}\text { Structural analysis of } \\
\text { civil and irrigation } \\
\text { structures }\end{array}$ & - & - & $\sqrt{ }$ & 6 & - & - & - & - \\
\hline 67 & Structural design I & - & - & - & - & - & - & $\sqrt{ }$ & 9 \\
\hline 68 & Structural design II & - & - & - & - & - & - & $\sqrt{ }$ & 9 \\
\hline 69 & Survey practical I & - & - & - & - & - & - & $\sqrt{ }$ & 9 \\
\hline 70 & Survey practical II & - & - & - & - & - & - & $\sqrt{ }$ & 9 \\
\hline 71 & Traffic engineering & $\sqrt{ }$ & 6 & - & - & - & - & - & - \\
\hline 72 & $\begin{array}{l}\text { Transportation } \\
\text { engineering I }\end{array}$ & - & - & - & - & - & - & $\sqrt{ }$ & 9 \\
\hline 73 & $\begin{array}{l}\text { Transportation } \\
\text { engineering II }\end{array}$ & - & - & - & - & - & - & $\sqrt{ }$ & 9 \\
\hline 74 & $\begin{array}{l}\text { Water supply } \\
\text { engineering }\end{array}$ & $\sqrt{ }$ & 9 & - & - & $\sqrt{ }$ & 6 & - & - \\
\hline & Total/Percentage & $\begin{array}{c}29(39.19 \% \\
)\end{array}$ & $\begin{array}{c}19 \\
2\end{array}$ & $\begin{array}{c}27(36.49 \% \\
)\end{array}$ & $\begin{array}{c}19 \\
8\end{array}$ & $\begin{array}{c}28(37.84 \% \\
)\end{array}$ & $\begin{array}{c}19 \\
7\end{array}$ & $\begin{array}{c}30(40.54 \% \\
)\end{array}$ & $\begin{array}{c}28 \\
1\end{array}$ \\
\hline
\end{tabular}

\subsection{Fundamental Courses for Bachelor degree}

Result in Table 4 shows that there are three fundamental courses taught in 3rd or 4th year of the Bachelor degree programme. These courses are entrepreneurship education, professional ethics and total quality management, where by DIT does not offer any course among the three courses. In that case, ATC and MUST offer one same course (Entrepreneurship education) which constitutes 33.33\% with 5 credits and 33.33\% with 6 credits respectively. However, SJCET offers two courses (professional ethics and total quality management) constituting $66.67 \%$ of the total fundamental courses. In this case, SJCET does not offer Entrepreneurship education course. These results predict similarities and differences of the contents of the curricula of Bachelor degree programme in the field of civil engineering. Thus, there is no consistency of fundamental courses offered by those institutions. Ebadi et al. (2020) compared the curricula of different Canadian universities and found that discrepancies exist between programmes, especially in number of courses offered.

Table 4. Fundamental Courses for Bachelor degree

\begin{tabular}{|c|c|c|c|c|c|c|c|c|c|}
\hline \multirow[b]{3}{*}{ SN } & \multirow[b]{3}{*}{ Fundamental courses } & \multicolumn{8}{|c|}{ Institutions } \\
\hline & & \multicolumn{2}{|c|}{ DIT } & \multicolumn{2}{|c|}{ ATC } & \multicolumn{2}{|c|}{ MUST } & \multicolumn{2}{|c|}{ SJCET } \\
\hline & & $\begin{array}{l}0 \\
\mathscr{n} \\
\vdots \\
\vdots\end{array}$ & 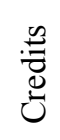 & 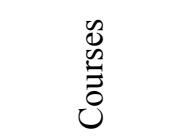 & 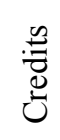 & 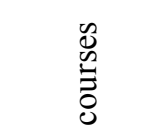 & $\begin{array}{l}\mathscr{\mathscr { Z }} \\
\stackrel{0}{0} \\
\ddot{U}\end{array}$ & 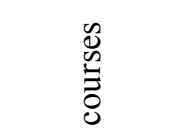 & 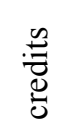 \\
\hline 1 & $\begin{array}{l}\text { Entrepreneurship } \\
\text { education }\end{array}$ & - & - & $\sqrt{ }$ & 5 & $\sqrt{ }$ & 6 & - & - \\
\hline 2 & Professional ethics & - & - & - & - & - & - & $\sqrt{ }$ & 6 \\
\hline 3 & Total quality management & - & - & - & - & - & - & $\sqrt{ }$ & 6 \\
\hline & Total/Percentage & $0(0 \%)$ & 0 & $1(33.33 \%)$ & 5 & $1(33.33 \%)$ & 6 & $2(66.67 \%)$ & 12 \\
\hline
\end{tabular}

\subsection{Core Courses for Bachelor degree}

Table 5 compares the core courses offered by the four institutions (DIT, ATC, MUST and SJCET) in the 3rd or 4th year of Bachelor degree. As a result, majority of these core courses 21(48.84\%) are offered at DIT with 177 credits. ATC has 15(34.88\%) core courses with 109 credits followed by MUST with 13 (30.23\%) core courses out of 43 core courses. However, in this case, SJCET has the lowest 6(13.95\%) core courses with 81 credits. This scenario of inconsistency of courses and credits in the curricula might be caused by several factors such as entry qualifications, duration of programmes, teaching and learning environment and, non-harmonized curricula among the institutions. Furthermore, these results correlate with the works of previous researchers (Ebadi et al., 
2020; Eshniyazov et al., 2020; Tabsh et al. 2012; Tapia and Najafi, 2003) who found inconsistency of student academic load, similarities and dissimilarities of courses of the same programme, semester system based programmes and, the existence of discrepancies between programmes especially in number of courses and credits.

Table 5. Core Courses for Bachelor degree

\begin{tabular}{|c|c|c|c|c|c|c|c|c|c|}
\hline \multirow[b]{3}{*}{$\mathrm{SN}$} & \multirow[b]{3}{*}{ Core courses } & \multicolumn{8}{|c|}{ Institutions } \\
\hline & & \multicolumn{2}{|c|}{ DIT } & \multicolumn{2}{|c|}{ ATC } & \multicolumn{2}{|c|}{ MUST } & \multicolumn{2}{|c|}{ SJCET } \\
\hline & & $\begin{array}{l}0 \\
0 \\
: \\
0 \\
0\end{array}$ & 异 & 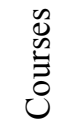 & 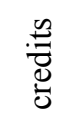 & $\begin{array}{l}\mathscr{D} \\
\stackrel{\mathscr{\omega}}{0} \\
0\end{array}$ & 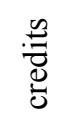 & 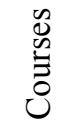 & : \\
\hline 1 & $\begin{array}{l}\text { Engineering } \\
\text { economics/and project } \\
\text { appraisal }\end{array}$ & $\sqrt{ }$ & 9 & $\sqrt{ }$ & 8 & $\sqrt{ }$ & 9 & - & - \\
\hline 2 & Structural steel design & $\sqrt{ }$ & 6 & - & - & $\sqrt{ }$ & 6 & - & - \\
\hline 3 & Waste water management & - & - & - & - & $\sqrt{1}$ & 6 & - & - \\
\hline 4 & Pavement maintenance & $\sqrt{ }$ & 6 & - & - & $\sqrt{ }$ & 6 & - & - \\
\hline 5 & $\begin{array}{l}\text { Construction of } \\
\text { multistory structures }\end{array}$ & - & - & - & - & $\sqrt{ }$ & 6 & - & - \\
\hline 6 & Building services & - & - & - & - & $\sqrt{ }$ & 6 & - & - \\
\hline 7 & Project I & - & - & $\sqrt{ }$ & 10 & $\sqrt{ }$ & 18 & $\sqrt{ }$ & 9 \\
\hline 8 & $\begin{array}{l}\text { Design of masonry and } \\
\text { retaining structures }\end{array}$ & - & - & - & - & $\sqrt{ }$ & 6 & - & - \\
\hline 9 & Structural timber design & $\sqrt{ }$ & 6 & - & - & $\sqrt{ }$ & 6 & - & - \\
\hline 10 & Solid waste management & $\sqrt{ }$ & 6 & - & - & $\sqrt{ }$ & 6 & - & - \\
\hline 11 & $\begin{array}{l}\text { Industrial building } \\
\text { construction }\end{array}$ & $\sqrt{ }$ & 6 & - & - & $\sqrt{ }$ & 6 & - & - \\
\hline 12 & Hydraulic structures & $\sqrt{ }$ & 6 & - & - & $\sqrt{ }$ & 6 & - & - \\
\hline 13 & Project II & - & - & $\sqrt{ }$ & 10 & $\sqrt{ }$ & 18 & $\sqrt{ }$ & 36 \\
\hline 14 & $\begin{array}{l}\text { Bridge design and } \\
\text { construction }\end{array}$ & $\sqrt{ }$ & 9 & - & - & - & - & - & - \\
\hline 15 & $\begin{array}{l}\text { Construction technology } \\
\text { services }\end{array}$ & $\sqrt{ }$ & 6 & - & - & - & - & - & - \\
\hline 16 & $\begin{array}{l}\text { Highway engineering } \\
\text { materials }\end{array}$ & $\sqrt{ }$ & 9 & - & - & - & - & - & - \\
\hline 17 & $\begin{array}{l}\text { Geographical information } \\
\text { systems (GIS) }\end{array}$ & $\sqrt{ }$ & 6 & - & - & - & - & - & - \\
\hline 18 & $\begin{array}{l}\text { Industrial practical } \\
\text { training }\end{array}$ & $\sqrt{ }$ & 12 & - & - & - & - & - & - \\
\hline 19 & Project data collection & $\sqrt{ }$ & 18 & - & - & - & - & - & - \\
\hline 20 & $\begin{array}{l}\text { Entrepreneurship for } \\
\text { engineers }\end{array}$ & $\sqrt{ }$ & 3 & - & - & - & - & - & - \\
\hline 21 & Foundation engineering & $\sqrt{ }$ & 9 & - & - & - & - & - & - \\
\hline 22 & $\begin{array}{l}\text { Masonry and retaining } \\
\text { wall design }\end{array}$ & $\sqrt{ }$ & 9 & - & - & - & - & - & - \\
\hline 23 & Project data analysis & $\sqrt{ }$ & 18 & - & - & - & - & - & - \\
\hline 24 & $\begin{array}{l}\text { Transportation } \\
\text { engineering }\end{array}$ & $\sqrt{ }$ & 9 & - & - & - & - & - & - \\
\hline 25 & $\begin{array}{l}\text { Pavement design and } \\
\text { construction }\end{array}$ & $\sqrt{ }$ & 9 & - & - & - & - & - & - \\
\hline 26 & Irrigation engineering & $\sqrt{ }$ & 6 & - & - & - & - & - & - \\
\hline 27 & Waste water engineering & $\sqrt{ }$ & 9 & - & - & - & - & - & - \\
\hline 28 & $\begin{array}{l}\text { Estimation and value } \\
\text { engineering }\end{array}$ & - & - & - & - & - & - & $\sqrt{ }$ & 9 \\
\hline 29 & $\begin{array}{l}\text { Economics and finance } \\
\text { for civil engineers }\end{array}$ & - & - & - & - & - & - & $\sqrt{ }$ & 9 \\
\hline 30 & $\begin{array}{l}\text { Construction } \\
\text { management }\end{array}$ & - & - & - & - & - & - & $\sqrt{ }$ & 9 \\
\hline
\end{tabular}




\begin{tabular}{|c|c|c|c|c|c|c|c|c|c|}
\hline \multirow[b]{3}{*}{$\mathrm{SN}$} & \multirow[b]{3}{*}{ Core courses } & \multicolumn{8}{|l|}{ Institutions } \\
\hline & & \multicolumn{2}{|l|}{ DIT } & \multicolumn{2}{|l|}{ ATC } & \multicolumn{2}{|l|}{ MUST } & \multicolumn{2}{|c|}{ SJCET } \\
\hline & & $\begin{array}{l}0 \\
0 \\
0 \\
0 \\
0\end{array}$ & 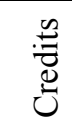 & 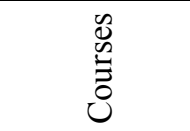 & 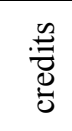 & 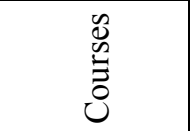 & : & 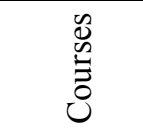 & : \\
\hline 31 & $\begin{array}{l}\text { Computer aided design } \\
\text { and drawing }\end{array}$ & - & - & - & - & - & - & $\sqrt{ }$ & 9 \\
\hline 32 & $\begin{array}{l}\text { Social and environmental } \\
\text { mgt. planning }\end{array}$ & - & - & $\sqrt{ }$ & 9 & - & - & - & - \\
\hline 33 & Contract management & - & - & $\sqrt{ }$ & 6 & - & - & - & - \\
\hline 34 & $\begin{array}{l}\text { Ground water } \\
\text { engineering }\end{array}$ & - & - & $\sqrt{ }$ & 9 & - & - & - & - \\
\hline 35 & $\begin{array}{l}\text { Reinforced cement } \\
\text { concrete design }\end{array}$ & - & - & $\sqrt{ }$ & 6 & - & - & - & - \\
\hline 36 & Site management & - & - & $\sqrt{ }$ & 6 & - & - & - & - \\
\hline 37 & $\begin{array}{l}\text { Water supply and public } \\
\text { health engineering }\end{array}$ & - & - & $\sqrt{ }$ & 9 & - & - & - & - \\
\hline 38 & $\begin{array}{l}\text { Maintenance engineering } \\
\text { and management }\end{array}$ & - & - & $\sqrt{ }$ & 6 & - & - & - & - \\
\hline 39 & Land use planning & - & - & $\sqrt{ }$ & 6 & - & - & - & - \\
\hline 40 & $\begin{array}{l}\text { Irrigation water } \\
\text { management }\end{array}$ & - & - & $\sqrt{ }$ & 6 & - & - & - & - \\
\hline 41 & $\begin{array}{l}\text { Design of concrete dams } \\
\text { and water tanks }\end{array}$ & - & - & $\sqrt{ }$ & 6 & - & - & - & - \\
\hline 42 & $\begin{array}{l}\text { Design of timber and } \\
\text { steel structures }\end{array}$ & - & - & $\sqrt{ }$ & 6 & - & - & - & - \\
\hline 43 & Procurement practice & - & - & $\sqrt{ }$ & 6 & - & - & - & - \\
\hline & Total /Percentage & $21(48.84 \%)$ & 177 & $15(34.88 \%)$ & 109 & $13(30.23 \%)$ & 105 & $6(13.95)$ & 81 \\
\hline
\end{tabular}

\subsection{Elective courses}

Results in Table 6 illustrate that in total there are 28 elective courses taught at 3rd or 4th year of the Bachelor degree programme. These elective courses include pre-stressed concrete design, irrigation engineering, bridge design and construction, transportation engineering, water resources management, coastal zone management and fundamentals of construction laws. Arguably, DIT has zero elective courses as compared to other institutions. However, this might be due to the fact that DIT has more core courses (21) as compared to ATC, MUST and SJCET respectively. On the other hand, SJCET has more elective courses 21(75\%) with 189 credits followed by MUST with $5(17.86 \%)$ elective courses constituting 30 credits. In this case, ATC has $3(10.71 \%)$ elective courses with 18 credits. According to Tapia and Najafi (2003) the major aspects taken into account in the curricula comparison were the duration of the programmes, courses of each curriculum and the differences in the student academic load. In fact, results illustrate the existing differences of the contents of the curricula of Bachelor degree in the field of civil engineering. Arguably, this can be due to the fact that the programmes of Bachelor of civil engineering has 6 or 8 semesters depending on the entry qualifications (Full Technician Certificate (FTC), Diploma or form six qualifications), with an average of seven modules per semester. Tabsh et al. (2012) assert that all the considered civil engineering programmes in the Arab region were based on the semester system. 
Table 6. Elective Courses for Bachelor degree

\begin{tabular}{|c|c|c|c|c|c|c|c|c|c|}
\hline \multirow[b]{3}{*}{ SN } & \multirow[b]{3}{*}{ Elective courses } & \multicolumn{8}{|c|}{ Tertiary Institutions (TI) } \\
\hline & & \multicolumn{2}{|c|}{ DIT } & \multicolumn{2}{|l|}{ ATC } & \multicolumn{2}{|l|}{ MUST } & \multicolumn{2}{|c|}{ SJCET } \\
\hline & & $\begin{array}{l}\text { क } \\
\overline{0} \\
\vdots \\
0\end{array}$ & 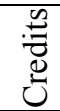 & 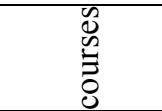 & 昜 & 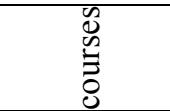 & 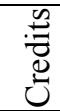 & 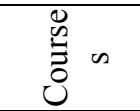 & 节 \\
\hline 1 & Pre-stressed concrete design & - & - & - & - & $\sqrt{ }$ & 6 & - & - \\
\hline 2 & Irrigation engineering & - & - & - & - & $\sqrt{ }$ & 6 & - & - \\
\hline 3 & Bridge design and construction & - & - & $\sqrt{ }$ & 6 & $\sqrt{ }$ & 6 & - & - \\
\hline 4 & Transportation engineering & - & - & - & - & $\sqrt{ }$ & 6 & - & - \\
\hline 5 & Water resources management & - & - & - & - & $\sqrt{ }$ & 6 & - & - \\
\hline 6 & Bridge Structures & - & - & - & - & - & - & $\sqrt{ }$ & 9 \\
\hline 7 & Storage Structures & - & - & - & - & - & - & $\sqrt{ }$ & 9 \\
\hline 8 & Design Of Plate And Shell Structure & - & - & - & - & - & - & $\sqrt{ }$ & 9 \\
\hline 9 & Tall Building & - & - & - & - & - & - & $\sqrt{ }$ & 9 \\
\hline 10 & Structural Dynamics & - & - & - & - & - & - & $\sqrt{ }$ & 9 \\
\hline 11 & Prefabricated Structures & - & - & - & - & - & - & $\sqrt{ }$ & 9 \\
\hline 12 & Wind Engineering & - & - & - & - & - & - & $\sqrt{ }$ & 9 \\
\hline 13 & Computer Aided Design Of Structures & - & - & - & - & - & - & $\sqrt{ }$ & 9 \\
\hline 14 & Pre-Stressed Concrete Structures & - & - & - & - & - & - & $\sqrt{ }$ & 9 \\
\hline 15 & Industrial Structures & - & - & - & - & - & - & $\sqrt{ }$ & 9 \\
\hline 16 & Smart Structures And Smart Materials & - & - & - & - & - & - & $\sqrt{ }$ & 9 \\
\hline 17 & Finite Element Techniques & - & - & - & - & - & - & $\sqrt{ }$ & 9 \\
\hline 18 & Ground Water Engineering & - & - & - & - & - & - & $\sqrt{ }$ & 9 \\
\hline 19 & Water Resources Engineering & - & - & - & - & - & - & $\sqrt{ }$ & 9 \\
\hline 20 & Management Of Irrigation Systems & - & - & - & - & - & - & $\sqrt{ }$ & 9 \\
\hline 21 & Coastal Zone Management & - & - & - & - & - & - & $\sqrt{ }$ & 9 \\
\hline 22 & Transportation Planning and Systems & - & - & - & - & - & - & $\sqrt{ }$ & 9 \\
\hline 23 & Traffic Engineering and Management & - & - & - & - & - & - & $\sqrt{ }$ & 9 \\
\hline 24 & Housing Planning and Design & - & - & - & - & - & - & $\sqrt{ }$ & 9 \\
\hline 25 & Railways And Airport Engineering & - & - & - & - & - & - & $\sqrt{ }$ & 9 \\
\hline 26 & Urban And Regional Development & - & - & - & - & - & - & $\sqrt{ }$ & 9 \\
\hline 27 & Constructed water wetlands & - & - & $\sqrt{ }$ & 6 & - & - & - & - \\
\hline 28 & Fundamentals of construction laws & - & - & $\sqrt{ }$ & 6 & - & - & - & - \\
\hline & Total /Percentage & 0 & 0 & $3(10.71 \%)$ & 18 & $5(17.86 \%)$ & 30 & $21(75 \%)$ & 189 \\
\hline
\end{tabular}

\subsection{Range of Courses and Credits in the Civil Engineering Curricula}

The courses of Bachelor of civil engineering programme were categorized into general courses, fundamental courses, core courses and elective courses as highlighted earlier in this study. Results in Figure 1 indicate that DIT was the 1 st institution that possesses more courses amounting to 83 courses with 599 credits. SJCET has 77 courses with 770 credits being the 2 nd in possession of more courses after DIT. However, MUST was the 3 rd having 73 courses with 504 credits. In this case, ATC was the lowest as it has 348 courses with 52 credits. Though the courses at these institutions are taken for three or four years, depending on the entry qualification of the candidate, results show that there was no uniformity among the courses offered by these institutions for the Bachelor degree programme. Furthermore, results indicate that SJCET has excessive academic load (770 credits) that students must carry as compared to academic loads at DIT (599), MUST (504 credits) and ATC (348 credits) respectively. On the other hand, students at ATC seem to be less stressed with respect to studies as they have moderate academic load (348 credits) that they must carry. This might be influenced by the lower number of classes that students must attend per week. This scenario at ATC may give room for students to participate in various extra curricula activities such as sports and other social activities. It is argued that, the parameter to measure the student load is the number of credits (Tapia and Najafi, 2003). However, the definition of credit differ from one institution to another. These study results correlate with previous researchers (Ebadi et al., 2020; Eshniyazov et al., 2020; Tabsh et al., 2012; Tapia and Najafi, 2003) who found inconsistency of student academic load, similarities and dissimilarities of courses of the same programme, semester system based programmes and, the existence of discrepancies between programmes especially in number of courses and credits. 


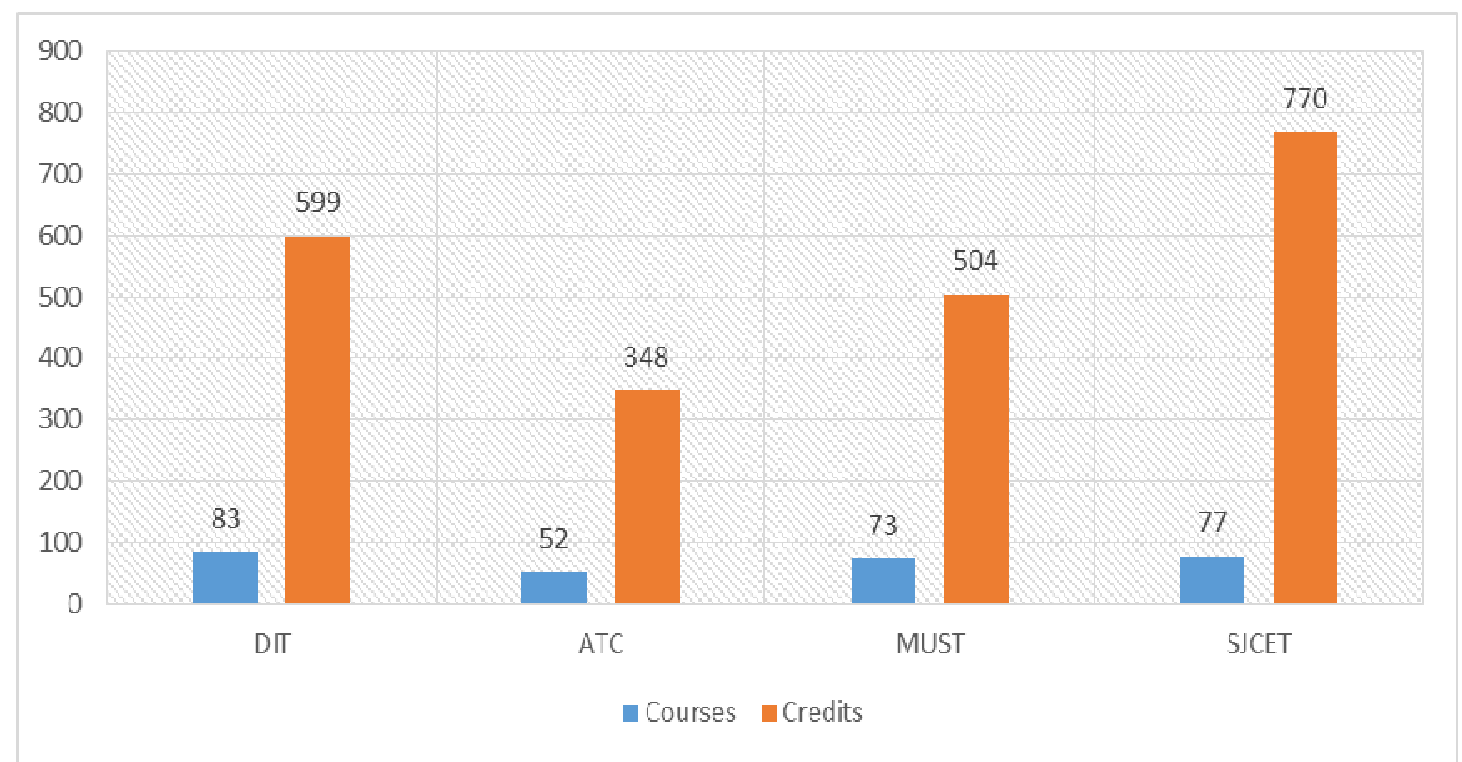

Figure 1. Range of Courses and Credits in the Civil Engineering Curricula

\section{Significance}

This article is of methodological and practical significance. The recommendations of the study would contribute improving quality of training Bachelor degree students in the field of civil engineering.

\section{Conclusion and recommendations}

The study was to examine the differences and similarities of the curricula for Bachelor degree programmes in the field of civil engineering offered by four institutions (DIT, ATC, MUST and SJCET) in view of improvement. It could be argued, in the sense that, the training of engineers requires continuous improvement to the content of the training curricula that provides relevant skills and knowledge. Therefore, it is imperative periodically to evaluate and revise the status of the curricula to address the needs of the professional changing trends in the labour market. The findings of the study indicated that DIT was the 1 st institution that possesses more courses amounting to 83 courses with 599 credits. SJCET has 77 courses with 770 credits being the 2nd in possession of more courses after DIT. However, MUST was the 3 rd having 73 courses with 504 credits. In this case, ATC was the lowest as it has 52 courses with 348 credits. Though the courses at these institutions are either taken for three or four years depending on the entry qualifications of the candidate, results indicate that there is no uniformity among the courses offered by these institutions for the Bachelor degree programme in the field of civil engineering. This scenario of inconsistency of courses and credits of the curricula offered by these institutions needs to be harmonized. It should be noted that, one of the major attributes of any good profession is a wellstructured intellectual content of the curriculum. In fact, this could help training institutions to produce competent demand-driven civil engineers who can compete equally in the labour market both nationally and internationally. Based on the study objective and results, the Authors recommends the following to practitioners, researchers and policies governing the curriculum of Bachelor degree programme:

- Training institutions should adopt harmonized teaching philosophy for undergraduate civil engineering students in view of producing knowledgeable and skilled civil engineers who can work independently and fit their jobs immediately after graduation.

- Institutions should provide curricula with harmonized number of credit-hours (academic load) for Bachelor degree students.

- There is a need to continuously monitor the content of the Bachelor degree programme in the field of civil engineering offered in all four institutions and adjust programme contents accordingly.

- There should be joint development of curricula of training programmes. This could help to minimize discrepancies of the curricula contents of the same programme offered in different institutions.

- Further research is suggested in developing an appropriate approach of capturing information related to curriculum review in all higher learning tertiary institutions. This could help to improve the curricula for bachelor degree programmes in the field of civil engineering offered in different institutions.

\section{Disclosure statement}

No potential conflict of interest with respect to the research, authorship, and/or publication of this article reported by the Authors. 


\section{Acknowledgement}

The Authors wish to gratefully acknowledge, with thanks, the agencies, institutions and individuals for their useful contributions to the study.

\section{References}

Akyazi, T., Alvarez, I., Alberdi, E., Oyarbide-Zubillaga, A., Goti, A., and Bayon, F. (2020). Skills Needs of the Civil Engineering Sector in the European Union Countries: Current Situation and Future Trends. Applied Science 10 (7226), pp.1-24.

Arusha Technical College. (2021).Prospectus. https://www.atc.ac.tz/images/downloads/2019 prospectus. Accessed: 29.04.2021.

Bhat $_{\mathrm{a}}$, S., Bhat $\mathrm{b}$, S., Raju, R., Souza, R., Binu, K. G. (2019). Collaborative Learning for Outcome Based Engineering Education: A Lean Thinking Approach. $9^{\text {th }}$ World Engineering Education Forum, WEEF Science Direct Procedia Computer Science, 00(2019) 000-000www.elsevier.com/locate/Procedia.

Chen, W.F., and Liew, J.Y. R. (2003). The Civil Engineering Handbook, 2nd ed. Washington: CRC Press.

Creswell, J.W. (2009). Research Design: Qualitative, Quantitative and Mixed Methods Approaches, SAGE Publications, London.

Dar es Salaam Institute of Technology. (2021). Prospectus. https://dit.ac.tz/documents/dit prospectus. Accessed: 29.04.2021.

Davies, E. A. J., and Marsh, C. M. (2021). "Canals and inland waterways". Encyclopedia Britannica, Invalid Date, https://www.britannica.com/technology/canal-waterway. Accessed 13 April 2021.

Ebadi, M. M., Richards, M., Brown, C., and Adeeb, S., (2020). Comparison of the civil engineering curriculum among several Canadian universities. Proceedings 2020 Canadian engineering education association (CEEA-ACEG20) Conference pp.1-7.

Eshniyazov, R., Bezzubko, B., Alimov, A., Arziev, A., Turdibaev, A., Pirnazarov, N. (2020). Bachelor Degree Programs In Building Materials Technology. European Journal of Molecular and Clinical Medicine 7(10), PP. 1780 .

Liu, F., and Siddharthan, R. V. (2016). The Differences between Undergraduate Education in Civil Engineering in China and America. American Journal of Educational Research 4(10), PP. 711-719. Available online at http://pubs.sciepub.com/education/4/10/1 C Science and Education Publishing DOI: 10.12691/education-4$10-1$.

MUST. (2021). Prospectus. https://must.ac.tz/portal/frontend/web/uploads/documents/15. Accessed: 29.04.2021.

NACTE. (2001). Accreditation and Recognition Regulations. Dar es salaam, Tanzania. https://www.nacte.go.tz/wp-content/uploads/2019/01/regulations-accreditation-and-recognition. Accessed: 29.04.2021.

Nwosu, O., Eyisi, G. U., Ekene, A. E. (2013). Comparative Study of Library and Information Science Curricula in Tertiary Institutions in South-East Nigeria. OSR Journal of Mechanical and Civil Engineering 5(5), PP. 33-41.

Oliver, R., Kersten, H., Vinkka-Puhakka, H., Alpasan, G., Bearn, D., Cema, I., Delap, E., Dummer, P., Goulet, J.P., Gugushe, T., Jeniati, E., Jerolimov, V., Kotsanos, N., Krifka, S., Levy, G., Neway, M., Ogawa, T., Saag, M., Sidlauskas, A., Skaleric, U., Vervoorn, M., White, D. (2008). Curriculum structure: principles and strategy. European Journal of Dent Education 12(suppl1), pp. 74-84. DOI: 10.1111/j.16000579.2007.00482.x

Serçe, F., and Acar, F. (2021). A comparative study of secondary mathematics curricula of Turkey, Estonia, Canada, and Singapore. Journal of Pedagogical Research 5(1), pp. 217-242. 2021http://dx.doi.org/10.33902/JPR.2021167798.

Shuaibu, H., Amin, S. H. M., Ismail, S. and Kamin, Y. (2019). Vocational colleges Curricula: a comparative study between Malaysia and Nigeria. Asian journal of interdisciplinary research 2(4), pp. 90-96.

SJCET. (2021). Prospectus. https://www.sjuit.ac.tz/SJUIT-Prospectus-2019-2020.pdf. Accessed: 29.04.2021.

$\mathrm{Su}, \mathrm{S}$. W. (2012). The Various Concepts of Curriculum and the Factors Involved in Curricula-making. Journal of Language Teaching and Research 3(1), pp. 153-158.

Sutopo, A. (2018).Translation Analysis on Civil Engineering Text Produced by Machine Translator. E3S Web of Conferences 31, pp. 2-5, https://doi.org/10.1051/e3sconf/20183110012ICENIS 2017.

Tabsh, S. W., Abdelfatah, A., Alhamaydeh, M., And Yehia, S. (2012). Comparison of Civil Engineering Curricula in the Arab World. International Journal of Engineering Education 28(5), pp. 1213-1220.

Tapia, P., and Najafi, F. T. (2003). A Comparison of the Civil Engineering Curriculum at the University of Florida and the Catholic University of the North, Chile. Proceedings of the 2003 American Society for Engineering Education Annual Conference and Exposition Copyright (C) 2003, American Society for Engineering Education, pp. 8.24.1- 8.24.7.

Tinsae, E. W. (2016). A critical Review and Analysis of the Definitions of Curriculum and the Relationship 
between Curriculum and Instruction. International Journal of Research in Engineering and Social Sciences 6(04), pp. 8-12.

Zhou, Y., Zhang, J., Li, L., Liao, S., and Fang, Z. (2014). Comparison Analysis of civil engineering undergraduate curriculum among universities in china, US and Europe. Journal of Applied Mechanics and Materials, 638-640, pp. 2402-2408. 